\title{
Evaluation of uncertainties in classical and component (blocked force) transfer path analysis (TPA)
}

\author{
A.T. Moorhouse ${ }^{1}$, J.W.R. Meggitt ${ }^{1}$, A.T. Elliott ${ }^{1}$ \\ ${ }^{1}$ Acoustics Research Centre, University of Salford, Greater Manchester, M5 4WT
}

\begin{abstract}
Transfer path analysis (TPA) has become a widely used diagnostic technique in the automotive and other sectors. In classic TPA, a two-stage measurement is conducted including operational and frequency response function (FRF) phases from which the contribution of various excitations to a target quantity, typically cabin sound pressure, are determined. Blocked force TPA (also called in situ Source Path Contribution Analysis, in-situ TPA and component TPA) is a development of the classic TPA approach and has been attracting considerable recent attention. Blocked force TPA is based on very similar two stage measurements to classic TPA but has two major advantages: there is no need to dismantle the vehicle and the blocked forces obtained are an independent property of the source component and are therefore transferrable to different assemblies. However, despite the now widespread reliance on classic TPA, and the increasing use of blocked force TPA in the automotive sector, it is rare to see any evaluation of the associated uncertainties. This paper therefore aims to summarize recent work and provide a guide to the evaluation of uncertainties in both forms of TPA. The various types of uncertainty are first categorized as, 'model', 'source' and 'experimental' uncertainties. Model uncertainties arise due to incomplete or inconsistent representation of the physical assembly by the measurements. Criteria are provided for evaluation of completeness in terms of measured quantities. Experimental and source uncertainties are evaluated through a first order propagation approach. Expressions are provided allowing the uncertainty in the target quantity to be estimated from measured quantities. Additional data storage and analysis is required but no additional measurements are needed over and above the usual TPA measurements. An illustrative example is provided.
\end{abstract}

\section{Introduction}

Transfer path analysis (TPA) has become a widely used diagnostic technique in the automotive industry, as well as in other industrial sectors [1]. The aim is to quantify the contributions of various structural transmission paths to a vibro-acoustic target quantity, typically cabin sound pressure. This breakdown provides valuable information for engineers to influence e.g. cabin sound pressure through modification of structural components. Noise sources considered include road noise, engine noise as well as others. Airborne sources and transmission can be included in TPA, but the focus here is on structural transmission. In this paper we consider the uncertainties and errors in two of the most widely used variants of TPA, namely classical $[2,3]$ (matrix inverse) and component (blocked force) TPA [4]. Other TPA variants, such as the mount stiffness method [1] and advanced TPA [5], are not considered although could be subject to a similar analysis.

In both classical and blocked force TPA, the assembled vehicle is delineated into a source region, $\mathrm{S}$, and a receiver region, $\mathrm{R}$ (see Figure 1). The source region contains all the source mechanisms of interest and frequently also includes some passive structures. For road noise for example, the source region includes the tire-road contact patch, the tire, wheel, hub and parts of the suspension such as wishbones. All parts of the vehicle downstream of the source-receiver interface are considered to belong to the receiver, which for road noise typically includes the cabin, body, perhaps subframes and possibly some upper parts of the suspension. It is assumed that a set of forces act on the receiver region from the source region. Normally in TPA these forces are considered to act at discreet points but may include moments as well as ordinary forces. The aim of TPA is to quantify the contributions of each of the forces to the target quantity, P. Arguably, these are contributions rather than 'transfer paths' but we will continue to use the term transfer path analysis since this name has become widely adopted.

\subsection{Measurements for TPA}

A two-stage measurement process is needed for both classical and blocked force TPA. The first phase is an operational test where the vehicle is run under appropriate load cycles while accelerations and sound pressures are recorded at various points on the structure and in the cabin. Essentially the same operational test is used for both classical and blocked force TPA. A 'passive' measurement phase is then conducted with the vehicle at rest in which frequency response functions (FRFs) are measured, typically using excitation from a hammer. In classical TPA, the source and receiver regions must be physically separated for this phase so that the FRFs relate purely to the receiver substructure. In blocked force TPA, the vehicle remains fully assembled and the FRFs therefore relate to the whole assembly.

The analysis also comprises two steps, the first an inverse step to calculate the forces acting on the source-receiver inter- 

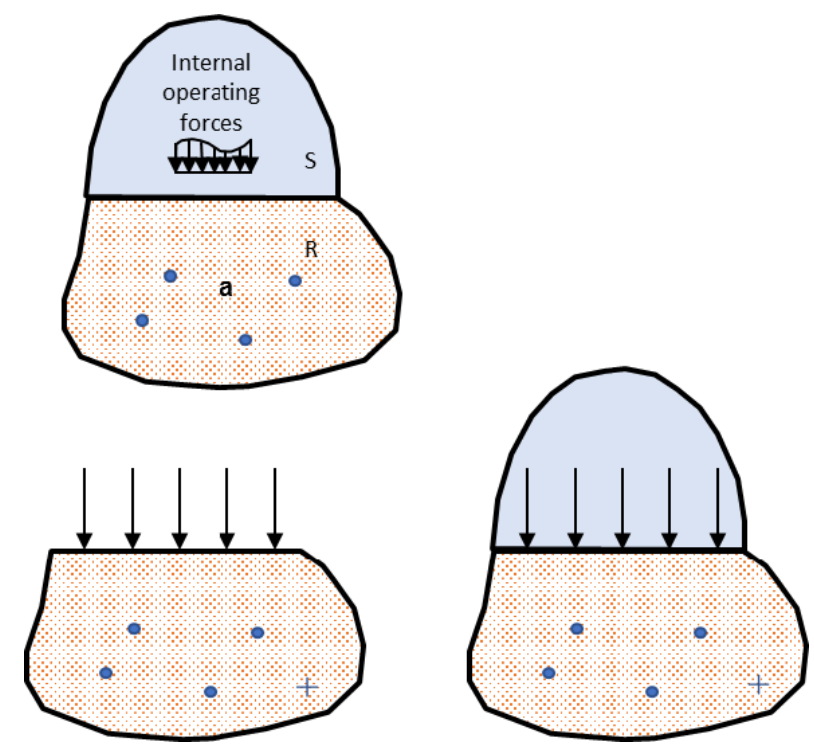

Figure 1: Schematic of measurement phases in TPA. Upper: operational test. Lower: FRF test for classical (left) and blocked force (right) TPA. S, R: source, receiver. $a$ : operational accelerations. Dots and crosses: responses at indicator and target locations.

face, and the second a forward or prediction step to calculate the contribution of these forces to the target quantity. The above measurement approaches can provide invaluable diagnostic information. However, they are known to be sensitive to measurement and other errors. It is perhaps surprising then that comparatively little attention has been devoted to the treatment of uncertainties in TPA. Hence, the aim of this paper is to provide a guide to the evaluation of uncertainties in both classical and blocked force TPA. The emphasis will be on providing as comprehensive a treatment as possible by bringing together already published results into a common framework. The reader will be referred to source publications for detailed derivations. In the following section we categorize the uncertainties affecting TPA as, 'model', 'source', 'or 'experimental' uncertainties. 'Model' uncertainties are then discussed by considering the 'completeness' and 'consistency' with which the measurements are able to represent the physical test structure. Treatment of 'source' and 'experimental' uncertainties are then discussed and practical methods for their evaluation are provided. These concepts are illustrated through a simulation example before a final summary and conclusions.

\section{Types of Uncertainty in TPA}

In this section we provide a categorization of the various forms of error and uncertainty affecting TPA. First though, we define the essential TPA equations.

\subsection{Classical and blocked force TPA}

The inverse and prediction steps in TPA are encapsulated respectively by the following equations:

$$
\mathbf{f}=\mathbf{Y}^{+} \mathbf{a}
$$

$$
\mathbf{p}=\mathbf{H f}
$$

In equation (1), $\mathbf{a}$, is a vector of operational accelerations measured at indicator positions, usually on or close to the sourcereceiver interface (see fig 1); $\mathbf{f}$ is an initially unknown vector of generalized forces acting at the interface degrees of freedom. $\mathbf{Y}$ is a matrix of FRFs relating the indicator and interface degrees of freedom and $\mathbf{Y}^{+}$indicates the inverse of this matrix (a pseudo-inverse for non-square matrices and a conventional inverse for square matrices). The force vector calculated from equation (1) is taken as the input to the prediction step outlined in equation (2) where $\mathbf{p}$ is a vector of (predicted) target quantities, usually cabin pressure at various points and $\mathbf{H}$ is a matrix of FRFs relating target and interface degrees of freedom. All quantities are assumed to be in the frequency domain and the dependence on radian frequency $\mathrm{W}$ is omitted for clarity.

Both classical and blocked force TPA can be described in terms of these same equations. However, there are some differences in the interpretation of the quantities apart from the indicator accelerations $\mathrm{A}$ in equation (1) which are the same for both methods, being based on identical measurements [4]. In classical TPA, the FRF measurements are conducted with the source substructure removed and hence the matrices $\mathbf{Y}$ and $\mathbf{H}$ relate to the receiver substructure only. In this case, the forces obtained from the inverse step, equation (1), are the contact forces at the interface, in other words the forces exerted by the source on the receiver. On the other hand, in blocked force TPA, the source and receiver substructures remain attached for the FRF measurements, so the matrices $\mathbf{Y}$ and $\mathbf{H}$ relate to the whole assembly. In this case, the forces obtained are the blocked forces of the source [6]. The physical interpretation of the blocked forces is slightly more abstract than for the contact forces, however, this minor disadvantage is compensated by a significant benefit in that they are independent of the receiver structure and can hence be transferred to assemblies where the same source is mounted on different receivers. This advantage is one of the main reasons why blocked force TPA is currently attracting interest. The inherent possibilities have been described in more detail elsewhere [1].

The 'output' of TPA depends on what it is being used for. As a diagnostic technique the desired quantities are the contributions to the target quantity, i.e. rewriting the righthand side of equation (2), the relative magnitudes of the terms in the sum (the transfer path contributions) are of primary interest:

$$
\mathbf{p}=\sum_{i} \mathbf{H}_{\mathbf{i}} f_{i}
$$

where $\mathbf{H}_{\mathbf{i}}$ are the columns of $\mathbf{H}$. However, with blocked force TPA there is the possibility of using the blocked forces purely for prediction purposes in which case the target quantity itself is of most interest. In this paper we will focus on the uncertainties in the target quantity, $\mathbf{p}$. The more detailed problem of estimating uncertainties in the transfer path contributions will be considered in a later paper. 


\begin{tabular}{ll} 
Category & Description \\
\hline \hline Model: & $\begin{array}{l}\text { Due to incomplete representation of all sig- } \\
\text { nificant forces at the source-receiver inter- } \\
\text { face } \\
\text { Due to variations in system properties } \\
\text { between the operational and passive test } \\
\text { phases }\end{array}$ \\
\hline Source & $\begin{array}{l}\text { Uncertainty inherent in the source opera- } \\
\text { tion }\end{array}$ \\
\hline
\end{tabular}

Experimental:

Measurement Due to noise in the measurement chain

Operator

Due to random errors in location and direction of forces during FRF measurement

\subsection{Categories of Uncertainty}

Meggitt et al.[7] have recently categorized the sources of uncertainty in inverse force identification as originating from the 'model', the 'source', and the 'experiment'. These categories have been slightly extended here for the context of TPA. The categories are summarized below and in table 1 and are described in more detail in the following two main sections.

'Model' uncertainty arises due to imperfect representation of the physical system by the measured data, for example due to inherent assumptions. A main cause is lack of 'completeness' in the representation of the interface, for example due to neglected degrees of freedom. Similarly, if the measurement points are not located at the actual location of the interface forces then an error can be expected. Strictly speaking, these are systematic errors rather than uncertainties but are an important reason (possibility the main reason) for poor results in TPA and so are included in this overview.

A further cause of 'model' error lies in lack of 'consistency' between the operational and FRF measurement phases, for example due to temperature variation or operating loads. Again, this is a systematic error rather than an uncertainty. Consistency is classed here as a form of model uncertainty, although it could be argued that it is a separate category.

'Source' uncertainties arise due to the variations (assumed random) in the operation of the source. Source uncertainties are handled here in a conventional way by obtaining a number of samples during operation from which estimates of statistical properties (mean and variance) are obtained. As an example, it is clear that road noise is a statistical source that requires repeated measurements over time with appropriate statistical treatment of the data.

'Experimental' uncertainties were defined by Meggitt et al [7] as comprising 'measurement' and 'operator' uncertainty. Measurement uncertainty consists of uncorrelated noise in the measurement chain which could originate from electrical noise in instruments or uncorrelated background noise in the acceleration signals, a. Again, this is handled by repeating measurements so as to obtain a set of samples from which statistical properties are derived. 'Operator' uncertainty arises due to a lack of repeatability in the measurement of FRFs. Assuming

that the structures are excited by an instrumented hammer during the FRF measurement phase, then differences (assumed to be random) are expected from one hit to the next both in the position and direction of the hit. (Note that differences in strength of the hit are not considered a source of uncertainty because, assuming linearity, the FRFs are normalized to the input force). It is normal practice to average the results of several hammer hits to obtain average FRFs, however, we recommend here that the individual FRFs obtained with each hit are retained so as to allow calculation of the variance (and covariance) in addition to the mean during postprocessing. Further details are provided later.

In the following we discuss first model uncertainty before then going on to present a framework for evaluation of all other types of uncertainty.

\section{Model Uncertainty}

An important cause of error in TPA lies in what we are terming 'model' uncertainty. Two categories of model uncertainty have been identified above, relating to completeness and consistency.

\subsection{Completeness}

Although TPA is an experimental technique, it is nevertheless necessary to adopt a 'model' of the physical system, comprising various assumptions, so that it can be represented by measured data. For TPA, a crucial aspect of the model is the representation of generalized forces at the source-receiver interface which are inevitably approximated or idealized to some extent. An important source of error in TPA, possibly the dominant source, is thought to be incomplete descriptions of the interface forces, for example if important degrees of freedom are neglected because of insufficient channel count, difficulties of access or difficulty in applying e.g. moments or in-plane forces for FRF measurement. For example, if there is significant excitation of the receiver by rotational degrees of freedom (moments) or in-plane forces at the interface then these forces need to be included in the force vector $\mathbf{f}$, which implies they must also be represented in the FRF matrices $\mathbf{Y}$ and $\mathbf{Y}$. An error will result if any are omitted.

The interface completeness criterion (ICC) has been developed to quantify the extent to which the chosen interface degrees of freedom provide a true representation the physical system. The essential concept, illustrated in figure (2), is that a force on one side of the interface will produce zero response on the other side if the interface is fully blocked. Blocking of the interface is not feasible physically but it can be achieved mathematically at the degrees of freedom included in the model, denoted, $c_{k}$, in figure (2). A non-zero response on the opposite side from the excitation is then taken to indicate the presence of unaccounted interface degrees of freedom, $c_{u}$ i.e. a lack of completeness in the interface description. 
The ICC is defined as follows [8]:

$$
C_{b a}=\frac{\left|\mathbf{Y}_{b \mathbf{a}}\left(\hat{\mathbf{Y}}_{b \mathbf{a}}\right)^{H}\right|^{2}}{\mathbf{Y}_{b \mathbf{a}}\left(\mathbf{Y}_{b \mathbf{a}}\right)^{H} \hat{\mathbf{Y}}_{b \mathbf{a}}\left(\hat{\mathbf{Y}}_{b \mathbf{a}}\right)^{H}}
$$

where $\mathbf{Y}_{b \mathbf{a}}$ is the FRF with excitation at a and response at $\mathrm{b}$ etc. (see below) and $\hat{\mathbf{Y}}_{b \mathbf{a}}=\mathbf{Y}_{b \mathbf{c}_{\mathbf{k}}} \mathbf{Y}_{\mathbf{c}_{\mathbf{k}} \mathbf{c}_{\mathbf{k}}}^{-1} \mathbf{Y}_{\mathbf{c}_{\mathbf{k}} \mathbf{a}}$. A complete interface corresponds to $C_{b a}=1$ and indicates that there are no neglected paths, i.e. $c_{u}=0$. At the other extreme, $C_{b a}=0$ indicates a totally incomplete interface description, in other words that none of the interface paths are included in the model, so $c_{k}=0 . C_{b a}$ has previously been termed the Interface Completeness Criterion (ICC) for consistency of terminology with, for example the Frequency Response Assurance Criterion (FRAC) [9] although both are more accurately 'coefficients' rather than 'criteria'.

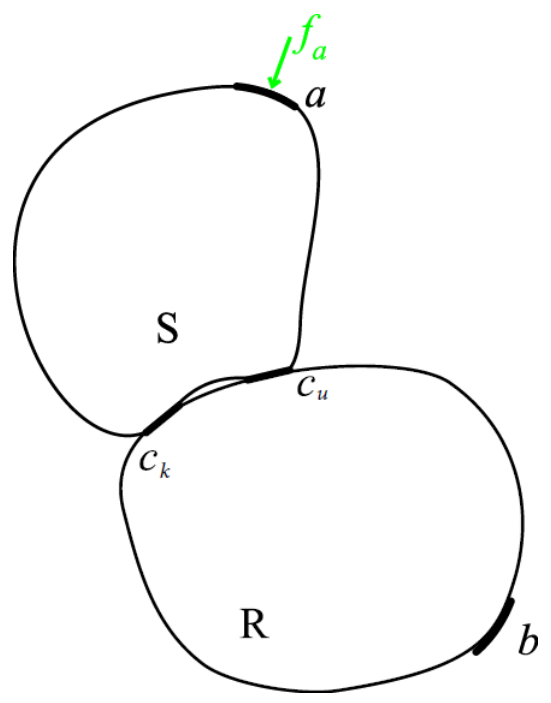

Figure 2: Illustration of the interface completeness criterion (ICC) concept: the interface is (mathematically) blocked at the known degrees of freedom, $c_{k}$; a non-zero response at $b$ on the receiver $\mathrm{R}$ due to excitation at $a$ on the source $\mathrm{S}$ then indicates the presence of neglected degrees of freedom at the interface, $c_{u}$, i.e. a lack of completeness in the model of the interface.

The FRFs needed for evaluation of the ICC, as defined in equation (3) are: $\mathbf{Y}_{b \mathbf{c}_{\mathrm{i}}}, \mathbf{Y}_{\mathbf{c}_{\mathbf{i}} \mathbf{c}_{\mathbf{i}}}^{-\mathbf{1}}$ and $\mathbf{Y}_{\mathbf{c}_{\mathbf{i}} \mathbf{a}}$. The ICC is equally valid for classical and blocked force TPA, however, since all FRFs required are measured with the source and receiver connected, then fewer additional measurements are required for blocked force TPA than for classical TPA:

1) $\mathbf{Y}_{b \mathbf{c}_{\mathbf{k}}}$ contains the accelerances with response at $b$ and excitation on the interface at $c_{k}$. These would normally be measured in blocked force TPA if at least some of the indicator positions are at $b$ (on the receiver away from the interface);

2) $\mathbf{Y}_{\mathbf{c}_{\mathbf{k}} \mathbf{c}_{\mathbf{k}}}^{-1}$ contains accelerances with response and excitation both on the interface at $c_{k}$. This would normally be measured in blocked force TPA if the indicator positions are at $c_{k}$ (on the interface);
3) $\mathbf{Y}_{\mathbf{c}_{\mathbf{k}} \mathbf{a}}$ contains the accelerances with excitation at locations $a$ on the source side of the interface and responses on the interface at $c_{k}$. This would not normally be measured, however, it is a fairly simple additional measurement since accelerometers will already be placed at $c_{k}$ in any case. This can be considered as a 'artificial excitation' of the source with the hammer.

An example of the ICC is given in figure (3) which indicates that the continuous interface between two halves of a plate is almost completely described by forces and moments at 10 discreet points on the interface (blue curve). However, when the moments are neglected the completeness is significantly reduced over certain frequency ranges (orange curve).

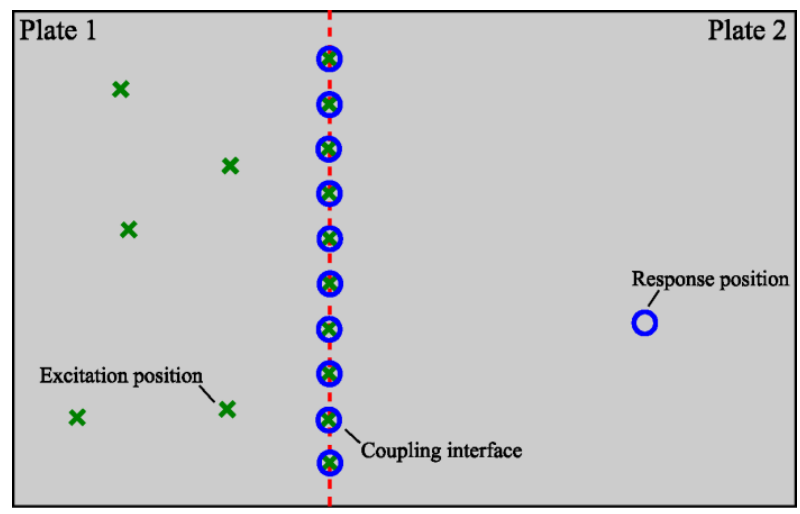

(a)

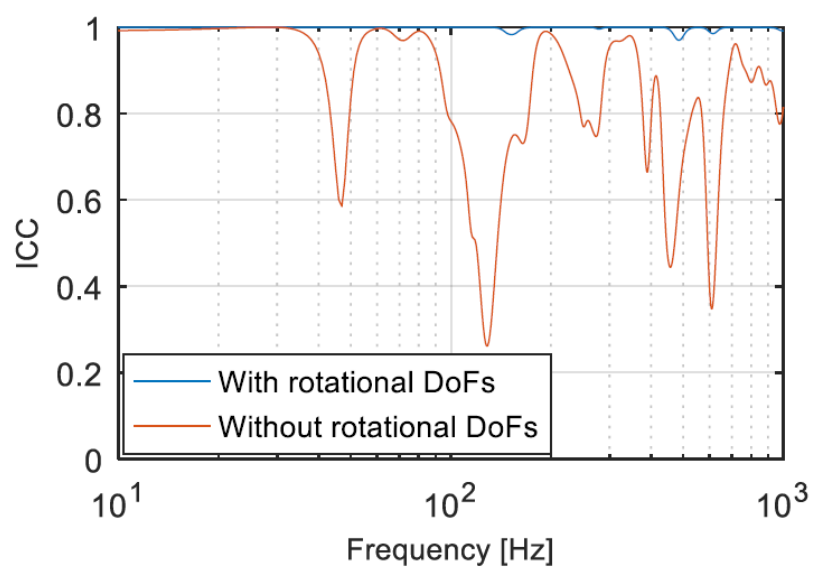

(b)

Figure 3: Illustration of the Interface completeness criterion (ICC). Top: "plate 1' and 'plate 2' are two sides of the same flat plate connected through a continuous interface. Continuous forces and moments along this interface are represented by generalized forces at 10 discreet points (the 'model'). Bottom: ICC with forces and moments included (blue); forces only (brown).

\subsection{Consistency}

As well as incompleteness, another cause of error in TPA occurs if there is inconsistency between the operational and FRF testing phases. For example, if there are temperature changes or if operating loads cause changes in the FRFs then then operational and FRF measurement actually describe slightly different 
systems. It was shown in $[10,11]$ that this can result in significant errors in certain frequency ranges. This can be illustrated by expressing the measured operational acceleration in terms of the 'true' force (or blocked force), $\mathbf{f}_{\text {true }}$, and the true FRF matrix, $\mathbf{Y}_{\text {true }}$.

$$
\mathbf{a}=\mathbf{Y}_{\text {true }} \mathbf{f}_{\text {true }}
$$

Note that the acceleration is measured directly and we do not have access to the terms on the right hand side so the 'true' FRF matrix, $\mathbf{Y}_{\text {true }}$, is implied in the acceleration measurements but is not obtained explicitly. When the inverse step is applied, as in equation (1), we pre-multiply by the inverse of the measured FRF matrix, Y:

$$
\mathbf{f}=\mathbf{Y}^{+}\left(\mathbf{Y}_{\text {true }} \mathbf{f}_{\text {true }}\right)
$$

Clearly, if there is consistency between the 'true' FRF matrix, $\mathbf{Y}_{\text {true }}$, and the measured one, $\mathbf{Y}$, then the FRF matrices in equation (5) cancel and the estimated forces (or blocked forces) $\mathbf{f}$ are equal to the true values. However, if there is any inconsistency then the cancellation will be incomplete. A typical scenario is when there is a shift in the frequency of a resonance or antiresonance, perhaps due to temperature or load changes; around the peak or trough, the FRF changes rapidly and a small shift in frequency can result in significant artefacts over a narrow frequency range due to lack of cancellation. Note that incompleteness in the model, as described above, is one possible cause of inconsistency. Ideally, it will be possible to quantify the consistency through a metric similar to the ICC developed above. However, this problem will be addressed in a later paper.

\section{Propagation of measurement uncertainties}

The aim of the uncertainty analysis outlined in this section is to quantify the uncertainties inherent in the estimates of the target quantity, p. In this section, we consider those uncertainties originating in the various TPA experiments (experimental uncertainty); we also consider source uncertainty since this may readily be evaluated as part of the same general approach. We start with a general framework for measurement uncertainties which is then applied to TPA. We go on to describe how the necessary inputs are obtained in the measurement phases and provide relationships for propagating the measured uncertainties through to the target quantity.

\subsection{Framework for measurement uncertainties}

Consider a general input-output system described by:

$$
\mathbf{y}=\mathbf{A} \mathbf{x}
$$

where $\mathbf{x}$ is an $n \times 1$ vector of inputs, $y$ an $m \times 1$ vector of outputs and $\mathbf{A}$ is the $m \times n$ system matrix. The problem is to propagate the uncertainties inherent in the quantities on the right hand side through to the outputs on the left hand side.

For uncertain inputs, $\mathbf{x}$, the covariance between any two elements of the output can be expressed using the law of error propagation as:

$$
\Sigma_{\mathbf{y}}=\mathbf{J}_{\mathbf{x}} \Sigma_{\mathbf{x}} \mathbf{J}_{\mathbf{x}}^{\mathbf{T}}
$$

where $\boldsymbol{\Sigma}_{\mathbf{y}}$ is the covariance matrix of the output and $\boldsymbol{\Sigma}_{\mathbf{x}}$ the covariance matrix of the input. For real inputs, $\boldsymbol{\Sigma}_{\mathbf{x}}$ is of dimension $n \times n$, but since the inputs in TPA will generally be complex this becomes $2 n \times 2 n$, since the real and imaginary parts must be described separately (as described later). Similarly, $\boldsymbol{\Sigma}_{\mathbf{y}}$ is of dimension $2 m \times 2 m$. $\mathbf{J}_{\mathbf{x}}$ is the Jacobian which is defined in terms of the derivatives of equation (6) with respect to each input and is of dimension (for complex inputs) $2 m \times 2 n$. In the following it will be described how to calculate the Jacobian from measured quantities. Further details about the derivation can be found in [7].

If, as well as the inputs, $\mathbf{x}$, the system matrix, $\mathbf{A}$, is also uncertain then additional terms are required to express the output covariance:

$$
\boldsymbol{\Sigma}_{\mathbf{y}}=\mathbf{J}_{\mathbf{x}} \boldsymbol{\Sigma}_{\mathbf{x}} \mathbf{J}_{\mathbf{x}}^{\mathbf{T}}+\mathbf{J}_{\mathbf{A}} \boldsymbol{\Sigma}_{\mathbf{A}} \mathbf{J}_{\mathbf{A}}^{\mathbf{T}}
$$

where $\boldsymbol{\Sigma}_{\mathbf{A}}$ is the covariance matrix for the uncertain system matrix which, for a complex matrix, has dimension $2 m n \times 2 m n$. $\mathbf{J}_{\mathbf{A}}$ is the Jacobian formed from the derivatives of equation (6) with respect to the elements of the system matrix and is of dimension $2 m \times 2 m n$.

Equation (8) assumes no correlation between the inputs and the system matrix which is a fair assumption for TPA since the operational and FRF tests are performed at different times. Despite this simplification, it is evident from equation (8) that a large number of elements are required to account for all possible cross correlations, particularly those between the elements of the system matrix. It is tempting to introduce further simplifying assumptions at this point, for example, the covariance matrix $\boldsymbol{\Sigma}_{\mathbf{A}}$ of the system matrix is diagonal if there is no correlation between the elements [12]. However, when using hammer measurements, it is usual to measure a full column of the matrix simultaneously in which case the elements within a column will be correlated to some extent. It has been shown that these correlations can have a significant influence on the output variances so the temptation to simplify will be resisted and all terms will be retained in the following analysis [12].

\subsection{General framework applied to TPA}

Having identified the general approach in the previous susbsection, we now proceed to apply this to the TPA problem. In effect, we apply the law of error propagation outlined in equation (8) to the forward and inverse steps of the TPA process as defined in equations (2) and (1) respectively.

\subsubsection{Forward (prediction) step}

Starting with the forward (prediction) step given in equation (2), it is noted that this is of the same form as equation (6). The law of error propagation defined in equation (8) can therefore be applied directly to give the covariance matrix of the target response (cabin sound pressure):

$$
\Sigma_{p}=\mathbf{J}_{\mathbf{f}} \Sigma_{\mathbf{f}} \mathbf{J}_{\mathbf{f}}^{\mathbf{T}}+\mathbf{J}_{\mathbf{H}} \boldsymbol{\Sigma}_{\mathbf{H}} \mathbf{J}_{\mathbf{H}}^{\mathbf{T}}
$$

where $\boldsymbol{\Sigma}_{\mathbf{f}}$ is the covariance matrix of the forces (or blocked forces for blocked force TPA) and $\boldsymbol{\Sigma}_{\mathbf{H}}$ the covariance matrix for the measured FRF matrix $\mathbf{H}$. $\mathbf{J}_{\mathbf{f}}$ and $\mathbf{J}_{\mathbf{H}}$ are the corresponding Jacobians. 


\begin{tabular}{|c|c|c|}
\hline Symbol & Description & How obtained \\
\hline$\Sigma_{\mathrm{a}}$ & $\begin{array}{l}\text { Covariance matrix of } \\
\text { measured operational } \\
\text { indicator responses }\end{array}$ & $\begin{array}{l}\text { Calculated from re- } \\
\text { peated measurement of } \\
\text { operational response } \\
\text { vector } \mathbf{a}\end{array}$ \\
\hline$\Sigma_{H}$ & $\begin{array}{l}\text { Covariance matrix of } \\
\text { measured FRF matrix, } \\
\mathbf{H}\end{array}$ & $\begin{array}{l}\text { Calculated from re- } \\
\text { peated measurement of } \\
\text { FRF matrix } \mathbf{H}\end{array}$ \\
\hline$\Sigma_{\mathbf{Y}}$ & $\begin{array}{l}\text { Covariance matrix of } \\
\text { measured FRF matrix, } \\
\mathbf{Y}\end{array}$ & $\begin{array}{l}\text { Calculated from re- } \\
\text { peated measurement of } \\
\text { FRF matrix Y }\end{array}$ \\
\hline $\mathbf{J}_{\mathbf{f}}$ & $\begin{array}{l}\text { Jacobian relating to } \\
\text { force vector, } \mathbf{f}\end{array}$ & See equation (24) \\
\hline $\mathbf{J}_{\mathbf{H}}$ & $\begin{array}{l}\text { Jacobian relating to } \\
\text { FRF matrix, } \mathbf{H}\end{array}$ & See equation (26) \\
\hline $\mathbf{J}_{\mathbf{a}}$ & $\begin{array}{l}\text { Jacobian relating to re- } \\
\text { sponse vector, a }\end{array}$ & See equation (27) \\
\hline $\mathbf{J}_{\mathbf{Y}}$ & $\begin{array}{l}\text { Jacobian relating to } \\
\text { FRF matrix, } \mathbf{Y}\end{array}$ & $\begin{array}{l}\text { See equation (28) for } \\
\text { square matrices and } \\
(29) \text { for non-square } \\
\text { matrices }\end{array}$ \\
\hline
\end{tabular}

Table 1: Quantities required for the calculation of measurement uncertainty in TPA.

The covariance matrix $\boldsymbol{\Sigma}_{\mathbf{p}}$ of the target responses are the quantities sought from this analysis. On the right hand side we have two covariance matrices, $\boldsymbol{\Sigma}_{\mathbf{f}}, \boldsymbol{\Sigma}_{\mathbf{H}}$ and two Jacobians, $\mathbf{J}_{\mathbf{f}}$ and $\mathbf{J}_{\mathbf{H}}$, which need to be evaluated before we can obtain this desired quantity. Of these, $\boldsymbol{\Sigma}_{\mathbf{H}}$ can be obtained from measured FRFs as described in [7, 13] and in the following. The Jacobians can also be described in terms of directly measured quantities as given in [13] and described later. The forces however are not measured directly and so an additional step is required to obtain their covariance matrix, $\Sigma_{\mathbf{f}}$, as described in the following.

\subsubsection{Inverse (force identification) step}

Equation (1) shows that the forces (or blocked forces), $\mathbf{f}$, are not measured directly but are inferred from other measured quantities in the inverse step of TPA. Applying the law of error propagation, equation (8), to equation (1) we obtain:

$$
\boldsymbol{\Sigma}_{\mathbf{f}}=\mathbf{J}_{\mathbf{a}} \boldsymbol{\Sigma}_{\mathbf{a}} \mathbf{J}_{\mathbf{a}}^{\mathbf{T}}+\mathbf{J}_{\mathbf{Y}^{+}} \boldsymbol{\Sigma}_{\mathbf{Y}^{+}} \mathbf{J}_{\mathbf{Y}^{+}}^{\mathbf{T}}
$$

where $\boldsymbol{\Sigma}_{\mathbf{a}}$ is the covariance matrix of the indicator responses, $\mathbf{a}$. In effect, these are the 'source' uncertainties. $\mathbf{J}_{\mathbf{a}}$ is the corresponding Jacobian. $\boldsymbol{\Sigma}_{\mathbf{Y}^{+}}$is the covariance matrix for the pseudo inverse of the measured FRF matrix $\mathbf{Y}^{+}$and $\mathbf{J}_{\mathbf{Y}^{+}}$the corresponding Jacobian.

Note that since the directly measured quantity is the FRF matrix $\mathbf{Y}$ rather than its pseudo inverse $\mathbf{Y}^{+}$, an additional step is required to propagate uncertainties of the measured FRFs through the matrix inversion. Fortunately, it is possible to rewrite equation (10) in a form more convenient for TPA as follows
[14]:

$$
\boldsymbol{\Sigma}_{\mathbf{f}}=\mathbf{J}_{\mathbf{a}} \boldsymbol{\Sigma}_{\mathbf{a}} \mathbf{J}_{\mathbf{a}}^{\mathbf{T}}+\mathbf{J}_{\mathbf{Y}} \boldsymbol{\Sigma}_{\mathbf{Y}} \mathbf{J}_{\mathbf{Y}}^{\mathbf{T}}
$$

where $\boldsymbol{\Sigma}_{\mathbf{Y}}$ is the covariance matrix for the (directly measured) FRF matrix, $\mathbf{Y} . \mathbf{J}_{\mathbf{Y}}$ is a correspondingly modified Jacobian which will be given later and again, is calculable from measured quantities. A derivation of equation (11) will be provided in [14]. Note that, whilst equation (8) provided an exact propagation of uncertainty (as equation (6) is linear), equation (11) provides only a first order approximation because the inverse of a matrix is non-linear function. The Jacobian $\mathbf{J}_{\mathbf{Y}}$ therefore provides a first order relation between $\mathbf{Y}$ and $\mathbf{Y}^{+}$and equation (11) is valid only in the presence of 'low levels' of FRF uncertainty.

Combining the results from this and the previous subsection we see that in order to calculate the desired variances of the target responses $\boldsymbol{\Sigma}_{\mathbf{p}}$ we require three covariance matrices and four Jacobians as summarized in Table 2. How to obtain these quantities will be shown in the following. However, first we briefly consider the issue of complex data.

\subsubsection{Handling of complex data}

In frequency domain TPA, as discussed here, all quantities will generally be complex. We therefore must consider the variance in both the real and the imaginary parts of each quantity and furthermore, the covariance between the real and imaginary parts. Therefore, in order to express the variance of a single complex quantity a $2 \times 2$ covariance matrix is required:

$$
\sigma_{x_{i} x_{j}} \rightarrow\left[\begin{array}{cc}
\sigma_{\mathfrak{R}\left(x_{i}\right) \mathfrak{R}\left(x_{j}\right)} & \sigma_{\mathfrak{R}\left(x_{i}\right) \mathfrak{I}\left(x_{j}\right)} \\
\sigma_{\mathfrak{I}\left(x_{i}\right) \mathfrak{R}\left(x_{j}\right)} & \sigma_{\mathfrak{I}\left(x_{i}\right) \mathfrak{I}\left(x_{j}\right)}
\end{array}\right]
$$

where $\sigma_{x_{i} x_{j}}$ is the covariance between elements $i$ and $j$ of a measured matrix or vector, and $\mathfrak{R}(\quad)$ and $\mathfrak{J}(\quad)$ represent real and imaginary parts respectively. For example, in the case of $i=$ $j, \sigma_{\mathfrak{R}\left(x_{i}\right) \mathfrak{R}\left(x_{i}\right)}$ is the variance of the real part, $\sigma_{\mathfrak{I}\left(x_{i}\right) \mathfrak{I}\left(x_{i}\right)}$ is that of the imaginary part and $\sigma_{\mathfrak{R}\left(x_{i}\right) \mathfrak{I}\left(x_{i}\right)}=\sigma_{\mathfrak{J}\left(x_{i}\right) \mathfrak{R}\left(x_{i}\right)}$ is the covariance between real and imaginary parts.

Fortunately, the required transformations can be handled relatively straightforwardly in the programming stage of analysis $[10,14,15]$ by the use of three complex matrix operators as defined below:

$$
\begin{aligned}
\mathbf{M}_{1}(A) & =\left[\begin{array}{c}
\mathfrak{R}(A) \\
\mathfrak{J}(A)
\end{array}\right] \\
\mathbf{M}_{2}(A) & =\left[\begin{array}{cc}
\mathfrak{R}(A) & -\mathfrak{J}(A) \\
\mathfrak{J}(A) & \mathfrak{R}(A)
\end{array}\right] \\
\mathbf{M}_{3}(A, B) & =\left[\begin{array}{cc}
\mathfrak{R}(A+B) & -\mathfrak{J}(A+B) \\
\mathfrak{J}(A+B) & -\mathfrak{R}(-A+B)
\end{array}\right]
\end{aligned}
$$

Each can be implemented in one line of code. For reference, the first, $\mathbf{M}_{\mathbf{1}}(\quad)$, applies to vectors, the second, $\mathbf{M}_{\mathbf{2}}(\quad)$, to analytic matrices satisfying the Cauchy Riemann equations and the third, $\mathbf{M}_{3}(\quad)$, to complex matrices not satisfying the CauchyRiemann equations. In the following, these operators will be applied elementwise. However, the same operations could potentially be applied to entire matrices, the important requirement is to ensure consistent ordering of matrix and vector ele- 
ments. The reader is referred to $[10,14,15]$ for further information on derivation and application.

\subsection{Obtaining the covariance matrices}

We now consider how the required covariance matrices are obtained from measurements. We start with the indicator response vector, a, before going on to treat the FRF matrices, $\mathbf{Y}$ and $\mathbf{H}$. Again, we stress the importance of correct and consistent ordering of all elements of all matrices and vectors.

\subsubsection{Covariance matrix of response vector, $\boldsymbol{\Sigma}_{\mathbf{a}}$}

In effect, the covariance matrix $\boldsymbol{\Sigma}_{\mathbf{a}}$ represents the source uncertainty, (although the measured values will to some extent be modified by the receiver structure to which the source is attached). To obtain the covariance matrix, the response vector, a (assumed $n \times 1$ ), is measured multiple times, say $k$ times in total. The resulting column vectors are arranged horizontally and the real part of each element is stacked on top of the corresponding imaginary part by elementwise application of the $\mathbf{M}_{\mathbf{1}}(\quad)$ operator (see equation 13a). The resulting measurement matrix is of dimension $2 n \times k$ :

$$
\overline{\mathbf{a}}=\left[\begin{array}{llll}
\mathbf{M}_{1}\left(\mathbf{a}^{(1)}\right) & \mathbf{M}_{1}\left(\mathbf{a}^{(2)}\right) & \cdots & \mathbf{M}_{1}\left(\mathbf{a}^{(k)}\right)
\end{array}\right]
$$

where $\mathbf{a}^{(i)}$ is the column vector of responses measured in the $i$ th measurement. For clarification, the expanded form of equation (14) is given by:

$$
\overline{\mathbf{a}}=\left[\begin{array}{cccc}
\mathfrak{R}\left(a_{1}{ }^{(1)}\right) & \mathfrak{R}\left(a_{1}{ }^{(2)}\right) & \ldots & \mathfrak{R}\left(a_{1}{ }^{(k)}\right) \\
\mathfrak{J}\left(a_{1}{ }^{(1)}\right) & \mathfrak{I}\left(a_{1}{ }^{(2)}\right) & \ldots & \mathfrak{I}\left(a_{1}{ }^{(k)}\right) \\
\mathfrak{R}\left(a_{2}{ }^{(1)}\right) & \mathfrak{R}\left(a_{2}{ }^{(2)}\right) & \ldots & \mathfrak{R}\left(a_{2}{ }^{(k)}\right) \\
\vdots & \vdots & \ddots & \vdots \\
\mathfrak{J}\left(a_{n}{ }^{(1)}\right) & \mathfrak{J}\left(a_{n}{ }^{(2)}\right) & \ldots & \mathfrak{I}\left(a_{n}{ }^{(k)}\right)
\end{array}\right]
$$

The covariance matrix is then obtained from equation (14) in the conventional manner using:

$$
\Sigma_{\mathbf{a}}=\frac{1}{k}\left[(\overline{\mathbf{a}}-\mathbb{E}(\overline{\mathbf{a}}))(\overline{\mathbf{a}}-\mathbb{E}(\overline{\mathbf{a}}))^{T}\right]
$$

where the expectation $\mathbb{E}(\quad)$ is taken along the rows.

\subsubsection{Covariance matrix of FRF matrix, $\boldsymbol{\Sigma}_{\mathbf{Y}}$}

In the evaluation of the FRF covariance matrices two additional factors arise that were not present for response vector, namely the need for multiple measurements and for reorganization of the FRF data.

The first point is that the FRF matrix, Y, must be recorded multiple times in order for the statistics to be evaluated. Whilst as described above, this is common practice for response measurements, it is not usual for FRF measurements to be processed in this way. In fact, the data is usually available because multiple measurements of FRFs are generally taken, however the individual results are typically discarded after averaging with no attempt to evaluate higher order statistics. However, by retaining the data from each measurement it is possible, as described below, to evaluate variance without resorting to any additional measurements. In the case of hammer measurements, this simply means that the results of each hit must be saved.

The second point is that, whereas FRF matrices are normally arranged such that the rows and columns correspond to response and excitation positions, for calculation of variance we require the correlations between every pair of matrix elements and a more convenient pattern is to arrange all the FRF elements into a single column vector. This can be achieved using a 'vectorization' operation, consisting of stacking the columns of the FRF matrix beneath each other moving left to right. This operation is simple to perform in many programming languages.

Considering the above, it is then possible to construct a measurement matrix similar to that for the response measurements defined in equation (14):

$$
\overline{\mathbf{Y}}=\left[\begin{array}{llll}
\mathbf{M}_{1}\left(\operatorname{vec}\left(\mathbf{Y}^{(1)}\right)\right) & \mathbf{M}_{\mathbf{1}}\left(\operatorname{vec}\left(\mathbf{Y}^{(2)}\right)\right) & \cdots & \mathbf{M}_{\mathbf{1}}\left(\operatorname{vec}\left(\mathbf{Y}^{(k)}\right)\right)
\end{array}\right]
$$

where, as with equation (14), the superscripts in brackets refer to the number of the measurement where $k$ is the total number of measurements made. vec( ) represents the vectorization operator as described above, thus, $\operatorname{vec}\left(\mathbf{Y}^{(i)}\right)$ represents the entire FRF matrix for measurement $i$ in vectorized form. $\mathbf{M}_{\mathbf{1}}(\quad)$ is the complex operator as defined in equation (13a) which again is applied elementwise.

Noting the similar form of equation (14) and (17) we can then express the covariance matrix in the same form as equation (16):

$$
\boldsymbol{\Sigma}_{\mathbf{Y}}=\frac{1}{k}\left[(\overline{\mathbf{Y}}-\mathbb{E}(\overline{\mathbf{Y}}))(\overline{\mathbf{Y}}-\mathbb{E}(\overline{\mathbf{Y}}))^{T}\right]
$$

where the expectation $\mathbb{E}(\quad)$ is taken along the rows.

\subsubsection{Covariance matrix of FRF matrix, $\mathbf{\Sigma}_{\mathbf{H}}$}

The covariance matrix for the target location FRFs, $\mathbf{H}$, can be treated in the same way as that for the indicator location FRFs, Y. It is simply necessary to replace $\mathbf{Y}$ with $\mathbf{H}$ in equations (17) and (18). The resulting equations are given below for completeness:

$$
\overline{\mathbf{H}}=\left[\begin{array}{llll}
\mathbf{M}_{\mathbf{1}}\left(\operatorname{vec}\left(\mathbf{H}^{(1)}\right)\right) & \mathbf{M}_{\mathbf{1}}\left(\operatorname{vec}\left(\mathbf{H}^{(2)}\right)\right) & \cdots & \mathbf{M}_{\mathbf{1}}\left(\operatorname{vec}\left(\mathbf{H}^{(k)}\right)\right)
\end{array}\right]
$$

and

$$
\Sigma_{\mathbf{H}}=\frac{1}{k}\left[(\overline{\mathbf{H}}-\mathbb{E}(\overline{\mathbf{H}}))(\overline{\mathbf{H}}-\mathbb{E}(\overline{\mathbf{H}}))^{T}\right]
$$

\subsection{Evaluating the Jacobians}

Having obtained the necessary covariance matrices from the previous section, the remaining quantities required from table 2 are the four Jacobians $\mathbf{J}_{\mathbf{a}}, \mathbf{J}_{\mathbf{f}}, \mathbf{J}_{\mathbf{Y}}, \mathbf{J}_{\mathbf{H}}$.

\subsubsection{General forms of Jacobian}

In order to illustrate the general form of the Jacobians, we return to the input-output problem outlined in equations (6) and (7). The Jacobian $\mathbf{J}_{\mathbf{x}}$ is defined in terms of the partial differen- 
tials of the outputs with respect to the uncertain inputs,

$$
\mathbf{J}_{\mathbf{x}}=\left[\begin{array}{cccc}
\frac{\partial y_{1}}{\partial x_{1}} & \frac{\partial y_{1}}{\partial x_{2}} & \cdots & \frac{\partial y_{1}}{\partial x_{n}} \\
\frac{\partial y_{2}}{\partial x_{1}} & \frac{\partial y_{2}}{\partial x_{2}} & \cdots & \frac{\partial y_{2}}{\partial x_{n}} \\
\vdots & \vdots & \ddots & \vdots \\
\frac{\partial y_{m}}{\partial x_{1}} & \frac{\partial y_{m}}{\partial x_{2}} & \cdots & \frac{\partial y_{m}}{\partial x_{n}}
\end{array}\right]
$$

For the simple example of equations (6) and (7) this results in $\mathbf{J}_{\mathbf{x}}=\mathbf{A}$.

If in addition to the input vector, the system matrix is uncertain, an additional term is needed as was introduced in equation (8). The corresponding Jacobian, $\mathbf{J}_{\mathbf{A}}$ is obtained by partial differentiation of the outputs with respect to each of the uncertain matrix entries:

$$
\mathbf{J}_{\mathbf{A}}=\left[\begin{array}{cccc}
\frac{\partial y_{1}}{\partial A_{11}} & \frac{\partial y_{1}}{\partial A_{21}} & \cdots & \frac{\partial y_{1}}{\partial A_{m n}} \\
\frac{\partial y_{2}}{\partial A_{11}} & \frac{\partial y_{2}}{\partial A_{21}} & \cdots & \frac{\partial y_{2}}{\partial A_{m n}} \\
\vdots & \vdots & \ddots & \vdots \\
\frac{\partial y_{m}}{\partial A_{11}} & \frac{\partial y_{m}}{\partial A_{21}} & \cdots & \frac{\partial y_{m}}{\partial A_{m n}}
\end{array}\right]
$$

This is a similar form to equation (21) except that the number of columns is increased to allow for partial differentiation by all $m n$ entries of the matrix. Thus, the dimension of $\mathbf{J}_{\mathbf{A}}$ is $m \times m n$ and it contains $m$ diagonal matrices arranged horizontally, each containing a repeated element of the vector $\mathbf{x}$.

$$
\begin{aligned}
\mathbf{J}_{\mathbf{A}} & =\left[\begin{array}{cccccccccc}
x_{1} & 0 & 0 & x_{2} & 0 & 0 & \cdots & \mathbf{x}_{\mathbf{n}} & 0 & 0 \\
0 & \ddots & 0 & 0 & \ddots & 0 & \cdots & 0 & \ddots & 0 \\
0 & 0 & x_{1} & 0 & 0 & x_{2} & \cdots & 0 & 0 & x_{n}
\end{array}\right] \\
& =\mathbf{x}^{\mathbf{T}} \otimes \mathbf{I} .
\end{aligned}
$$

The compact form of notation on the righthand side introduces the Kronecker product, $\otimes$, where every term of the matrix or vector on the left of the symbol is multiplied by the matrix or vector on the right. Fortunately, the Kronecker product is a standard function in many programming languages which facilitates implementation of the above.

\subsubsection{Jacobians required for TPA}

We now present expressions for the Jacobians required in TPA starting with those for the prediction step outlined in equation (2). We note that equation (2) is of the same form as equation (6), so the Jacobian $\mathbf{J}_{\mathbf{f}}$ from equation (9) can be written as:

$$
\mathbf{J}_{\mathbf{f}}=\mathbf{M}_{\mathbf{2}}(\mathbf{H})
$$

where the complex matrix operator $\mathbf{M}_{\mathbf{2}}(\quad$ ) (equation (10b)) has been introduced to deal separately with the real and imaginary parts. The second Jacobian from equation (9) is of a similar form to equation (23), so:

$$
\mathbf{J}_{\mathbf{f}}=\mathbf{M}_{2}\left(\mathbf{f}^{\mathbf{T}} \otimes \mathbf{I}\right)
$$

This can be rewritten in terms of directly measured quantities by substituting in equation (1), giving:

$$
\mathbf{J}_{\mathbf{f}}=\mathbf{M}_{\mathbf{2}}\left(\left(\mathbf{Y}^{+} \mathbf{a}\right)^{T} \otimes \mathbf{I}\right)
$$

where, again, $\mathbf{M}_{\mathbf{2}}(\quad)$ has been applied to deal with real and imaginary parts.

We now consider the inverse step outlined in equation (1), for which a similar analysis can be applied, albeit slightly complicated by the presence of the pseudo inverse. The first term of equation (11) is similar to that of equation (9) which leads to:

$$
\mathbf{J}_{\mathbf{a}}=\mathbf{M}_{\mathbf{2}}\left(\left(\mathbf{Y}^{+}\right)\right.
$$

The remaining Jacobian, $\mathbf{J}_{\mathbf{Y}}$, requires a more involved analysis because of the pseudo inverse and the result is presented here without derivation; the reader is referred to [14] for further details. For square matrices $\mathbf{J}_{\mathbf{Y}}$ takes the following form:

$$
\mathbf{J}_{\mathbf{Y}}=\mathbf{M}_{\mathbf{2}}\left(\left(-\mathbf{Y}^{-\mathbf{1}} \mathbf{a}\right)^{T} \otimes \mathbf{Y}^{-\mathbf{1}}\right)
$$

However, when the matrix is non-square, which will often be the case, a lengthier expression is needed using the third form of the complex matrix operator from equation (13c):

$$
\begin{array}{r}
\mathbf{J}_{\mathbf{Y}}=\mathbf{M}_{\mathbf{3}}\left(\left(-\mathbf{Y}^{-\mathbf{1}} \mathbf{a}\right)^{T} \otimes \mathbf{Y}^{-\mathbf{1}}\right),\left(\left[\left(\left(\mathbf{I}-\mathbf{Y} \mathbf{Y}^{+}\right) \mathbf{a}\right)^{T} \otimes \mathbf{Y}^{+} \mathbf{Y}^{+\mathbf{H}}\right]+\cdots\right. \\
\left.\cdots\left[\left(\mathbf{Y}^{+} \mathbf{Y}^{+\mathbf{H}} \mathbf{a}\right)^{T} \otimes\left(\mathbf{I}-\mathbf{Y} \mathbf{Y}^{+}\right)\right] \mathbf{K}\right)
\end{array}
$$

where $\mathbf{K}$ is known as a commutation matrix and gives a relation between $\mathbf{K} v e c(\mathbf{A})=\operatorname{vec}\left(\mathbf{A}^{\mathbf{T}}\right)$ [16]. $\mathbf{K}$ is uniquely defined by the dimensions of $\mathbf{A}$. For example, if $\mathbf{A}$ is of dimensions $3 \times 2, \mathbf{K}$ is given by,

$$
\mathbf{K}=\left[\begin{array}{llllll}
1 & 0 & 0 & 0 & 0 & 0 \\
0 & 0 & 0 & 1 & 0 & 0 \\
0 & 1 & 0 & 0 & 0 & 0 \\
0 & 0 & 0 & 0 & 1 & 0 \\
0 & 0 & 1 & 0 & 0 & 0 \\
0 & 0 & 0 & 0 & 0 & 1
\end{array}\right]
$$

All of the elements necessary for evaluation of the source and experimental uncertainties are now available. The procedure is summarized in the following.

\subsection{Summary for source and experimental uncertainties}

The procedure for calculating uncertainties can be summarized by substituting equation (11) into equation (9):

$$
\Sigma_{\mathbf{p}}=\mathbf{J}_{\mathbf{f}}\left(\mathbf{J}_{\mathbf{a}} \boldsymbol{\Sigma}_{\mathbf{a}} \mathbf{J}_{\mathbf{a}}^{\mathbf{T}}+\mathbf{J}_{\mathbf{Y}} \boldsymbol{\Sigma}_{\mathbf{Y}} \mathbf{J}_{\mathbf{Y}}^{\mathbf{T}}\right) \mathbf{J}_{\mathbf{f}}^{\mathbf{T}}+\mathbf{J}_{\mathbf{H}} \boldsymbol{\Sigma}_{\mathbf{H}} \mathbf{J}_{\mathbf{H}}^{\mathbf{T}}
$$

The following steps are then required:

1) $\Sigma_{\mathbf{a}}$ is evaluated by repeated measurement of the operational response vector, a, and calculation of the covariance matrix according to equations $(14,16)$;

2) $\Sigma_{\mathbf{Y}}$ is evaluated by repeated measurement of the FRF matrix, $\mathbf{Y}$, during the passive measurement phase and calculation of the covariance matrix according to equations $(17,18)$; 


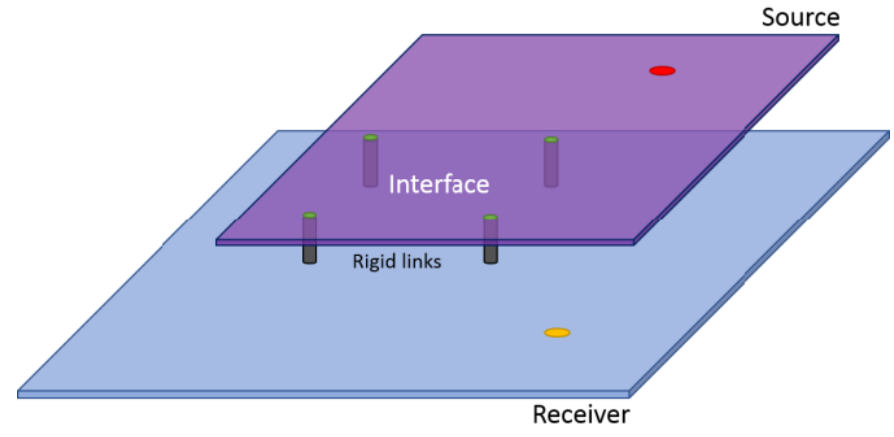

Figure 4: System for numerical example. The source and receiver are both flat plates. The red dot is the location of the 'internal' operating forces of the source (not normally accessible). The target response, $\mathbf{p}$, is evaluated at the yellow dot and indicator responses, a, at the interface locations (green). The FRF matrix, $\mathbf{Y}(4 \times 4)$, is evaluated at the interface locations (green) and $\mathbf{H},(1 \times 4)$ between the interface and target locations.

3) $\boldsymbol{\Sigma}_{\mathbf{Y}}$ is evaluated in a similar fashion to $\mathbf{Y}$ using equations $(19,20)$;

4) The Jacobians $\mathbf{J}_{\mathbf{a}}, \mathbf{J}_{\mathbf{f}}, \mathbf{J}_{\mathbf{Y}}, \mathbf{J}_{\mathbf{H}}$ are evaluated using equations $(24,26,27,28)$;

5) Values for all terms are substituted into equation (31).

\subsubsection{Correlation between $\mathbf{Y}$ and $\mathbf{H}$}

Note that equation (27) and all the preceding analysis assumes there is no correlation between the indicator position FRFs, Y, and the target position FRFs, H. This is a reasonable assumption if they are obtained in separate tests, which would occur for reciprocal measurement for example with a volume velocity source. However, if forward FRF measurement is used, with structural excitation at the interface then it is possible to obtain responses at both indicator and target locations simultaneously, in which case there will be correlation within the columns of $\mathbf{H}$ and $\mathbf{Y}$ and an additional term is required in equation (27):

$$
\Sigma_{\mathrm{p}}=\mathbf{J}_{\mathbf{f}}\left(\mathbf{J}_{\mathbf{a}} \Sigma_{\mathbf{a}} \mathbf{J}_{\mathbf{a}}^{\mathrm{T}}+\mathbf{J}_{\mathbf{Y}} \Sigma_{\mathbf{Y}} \mathbf{J}_{\mathbf{Y}}^{\mathrm{T}}\right) \mathbf{J}_{\mathbf{f}}^{\mathbf{T}}+\mathbf{J}_{\mathbf{H}} \boldsymbol{\Sigma}_{\mathbf{H}} \mathbf{J}_{\mathbf{H}}^{\mathrm{T}}+2 \mathbf{J}_{\mathbf{H Y}} \boldsymbol{\Sigma}_{\mathbf{H Y}} \mathbf{J}_{\mathrm{HY}}^{\mathrm{T}}
$$

Based on analysis in [13], the most likely effect of neglecting this correlation is a significant overestimation of the overall uncertainty. Since the estimates are likely to be on the safe side a full analysis of this effect will be left to a later paper. However, it is worth mentioning that there are potentially important implications for whether forward or reciprocal measurement of FRFs provides the most reliable results in TPA.

\section{Numerical Example}

In this section, the estimation of uncertainties in TPA is illustrated by a numerical example. Measurement examples of certain aspects of the above analysis are also provided in [7, 13]. The system evaluated is shown in figure (4). The source and receiver are both flat plates, connected at the top of 4 rigid connections. The lack of repeatability in the hammer hits for the
FRF measurements is simulated by randomly varying the excitation position which leads to an ensemble of measurements with the spread shown in the upper plot of figure (5). The FRF covariance matrices, $\boldsymbol{\Sigma}_{\mathbf{Y}}$ and $\boldsymbol{\Sigma}_{\mathbf{H Y}}$ are evaluated from this ensemble. The FRF variance has been selected for illustration purposes to lie on the high side of what would be expected from a typical physical measurement. The receiver response is contaminated by noise as shown in the lower plot of figure (5) leading to estimates of response variance, $\boldsymbol{\Sigma}_{\mathbf{a}}$.

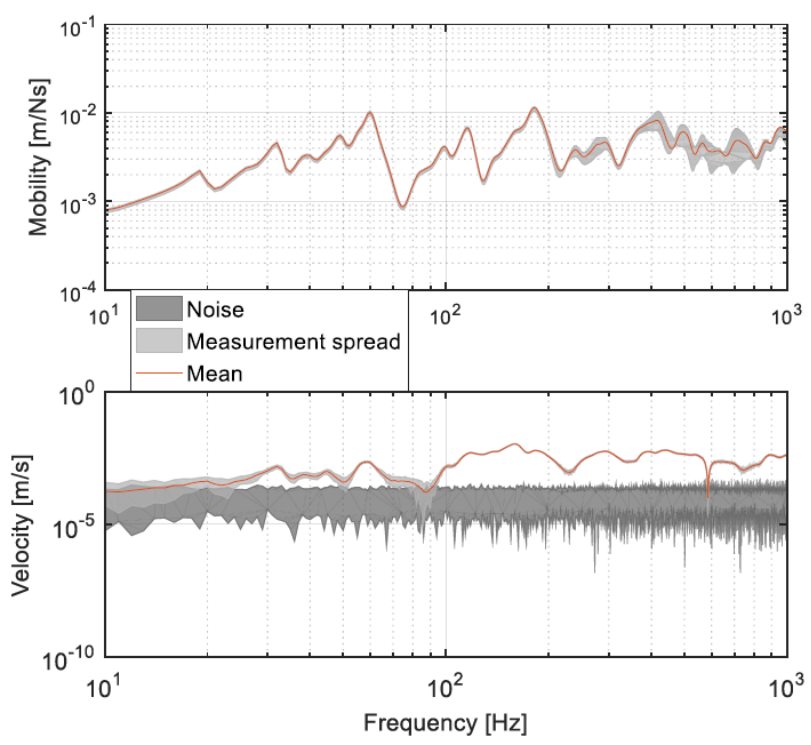

Figure 5: Upper: example mean FRF with spread of results from different hammer hits. Lower: example receiver response showing the spread of results with added noise.
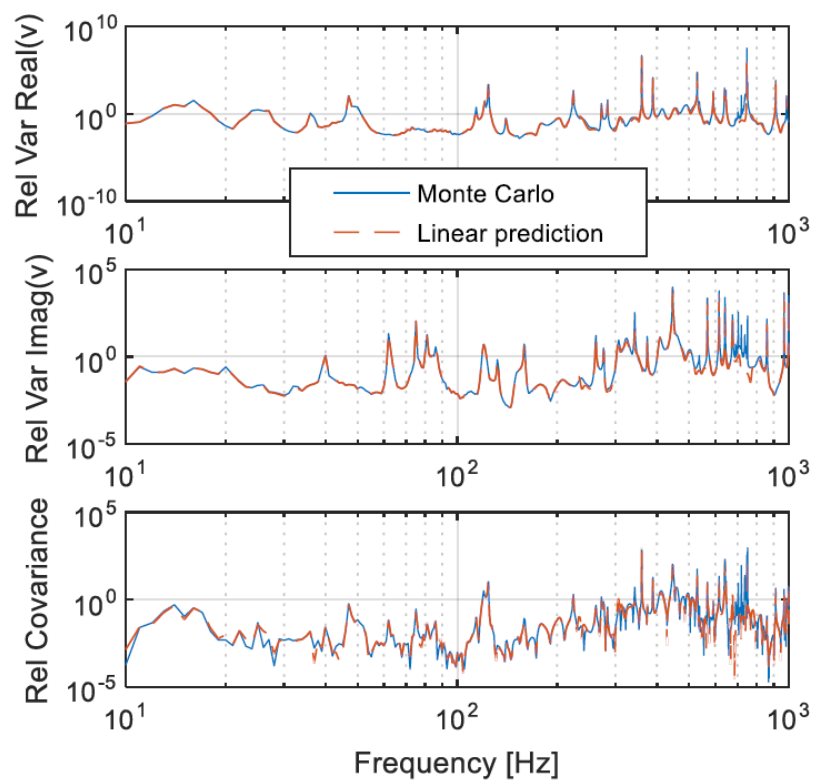

Figure 6: Bivariate uncertainty of the predicted response (compared against a Monte-Carlo solution)

Figure (6) shows the relative variance for the real and imag- 
inary parts of the target response as predicted from the above calculation scheme. Note that the covariance between the real and imaginary parts is significant and cannot be neglected. Also shown are the results from a Monte-Carlo analysis of the same data. Results indicate that the first order analysis described earlier provides a good estimate of uncertainties in the output, at least for the level of input uncertainty level considered (believed to be realistic).

Figure (7) shows the predicted magnitude of the target response overlaid with the $95 \%$ confidence intervals obtained from the above analysis. These confidence intervals were obtained assuming the real and imaginary parts of the response were joint normally distributed. A Monte-Carlo method was then used to estimate the confidence bounds for the magnitude of the response [13]. It can be seen that for this example the variance at low frequencies $(; 50 \mathrm{~Hz})$ primarily derives from background noise in the indicator responses. However, at high frequencies $(300-600 \mathrm{~Hz})$ the main source is the lack of repeatability in the FRF measurements.

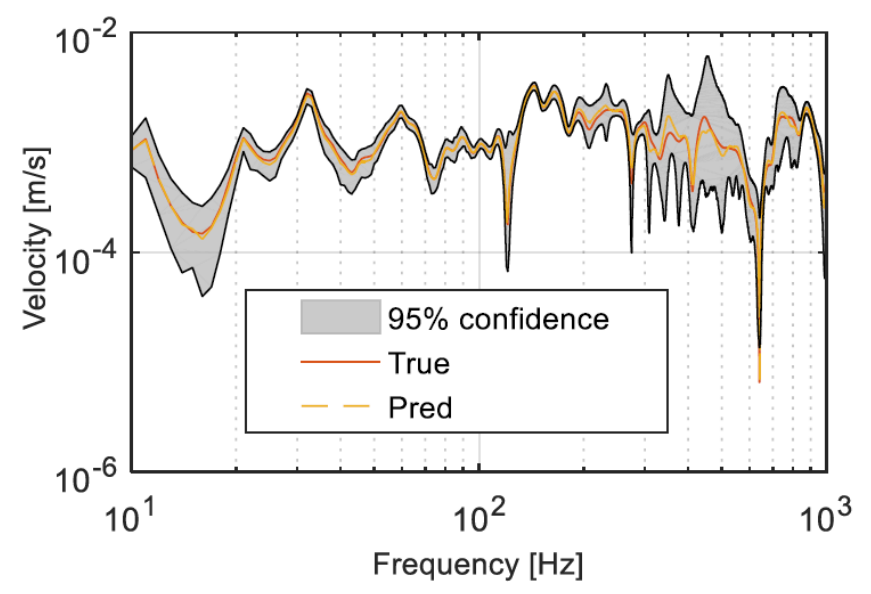

Figure 7: Predicted magnitude of the target response with a 95\% confidence bounds

\section{Summary/Conclusions}

The uncertainties affecting TPA measurements have been categorized as: model, source and experimental. Model uncertainties occur due to incompleteness or inconsistency in the representation of the physical system by the measurements and are a significant potential cause of error. The Interface Completeness Criterion (ICC) has been introduced as a way of quantifying the (lack of) completeness. A corresponding metric for consistency is under development.

Source and experimental uncertainties are random variations. A first order framework for estimating the influence of these input uncertainties on the target quantity in both classical and blocked force TPA has been outlined and illustrated by a numerical example. Three covariance matrices are required for the input quantities, i.e. the indicator accelerations and the FRFs for indicator and target locations. In addition, four Jacobian matrices are required which can all be calculated from directly measured quantities. No additional measurements are required over and above the usual TPA measurements, although additional data storage and calculation is required.

\section{References}

[1] M.V. van der Seijs, D.D. Klerk, and D.J. Rixen. General framework for transfer path analysis: History, theory and classification of techniques. Mechanical Systems and Signal Processing, pages 1-28, 2015.

[2] J.W. Verheij. Multi-path sound transfer from resiliently mounted shipboard machinery. PhD thesis, TNO, 1982.

[3] B J Dobson and E Rider. A review of the indirect calculation of excitation forces from measured structural response data. Proceedings of the Institution of Mechanical Engineers, Part C: Journal of Mechanical Engineering Science 1989-1996 (vols 203-210), 204(23):69-75, 1990.

[4] A. S. Elliott, A. T. Moorhouse, T. Huntley, and S. Tate. In-situ source path contribution analysis of structure borne road noise. Journal of Sound and Vibration, 332(24):6276-6295, 2013.

[5] F.X. Magrans. Method of measuring transmission paths. Journal of Sound and Vibration, 74(3):321-330, 1981.

[6] A.T. Moorhouse, A.S. Elliott, and T.A. Evans. In situ measurement of the blocked force of structure-borne sound sources. Journal of Sound and Vibration, 325(4-5):679-685, sep 2009.

[7] J.W.R. Meggitt, A.S. Elliott, and A.T. Moorhouse. A covariance based framework for the propagation of uncertainty through inverse problems with an application to force identification. Mechanical Systems and Signal Processing, (124):275-297, 2019.

[8] J.W.R. Meggitt, A.T. Moorhouse, and A.S. Elliott. On the problem of describing the coupling interface between sub-structures : an experimental test for 'completeness'. In IMAC XVIII, pages 1-11, Orlando, 2018.

[9] R.J. Allemang. The Modal Assurance Criterion (MAC): Twenty Years of Use and Abuse. In Proceedings of SPIE - The International Society for Optical Engineering, volume 4753, pages 397-405, 2002.

[10] J.W.R. Meggitt, A.S. Elliott, A.T. Moorhouse, A. Clot, and R.S. Langley. Development of a Hybrid FE-SEA-Experimental Model: Experimental Subsystem Characterisation. In NOVEM: Noise and Vibration - Emerging Methods, Ibiza, 2018.

[11] A. Clot, R. S. Langley, J.W.R. Meggitt, A.T. Moorhouse, and A.S. Elliott. Development of a Hybrid FE-SEA-Experimental Model: Theoretical Formulation. In NOVEM: Noise and Vibration - Emerging Methods, Ibiza, 2018.

[12] J.W.R. Meggitt. On the treatment of uncertainty in experimentally measured frequency response functions. Metrologia, 55:806-818, 2018.

[13] J.W.R. Meggitt and A.T. Moorhouse. A Combined Framework for the Propagation of Uncertainty in Transfer Path Analysis. (In Preperation).

[14] J.W.R. Meggitt, A.S. Elliott, A.T. Moorhouse, G. Banwell, H. Hopper, and J. Lamb. Broadband characterisation of in-duct acoustic sources using an equivalent source approach. Journal of Sound and Vibration, 2018.

[15] J.W.R. Meggitt and A.T. Moorhouse. A covariance based framework for the propagation of correlated uncertainty in frequency based dynamic sub-structuring. Mechanical Systems and Signal Processing (Submitted), 2019.

[16] A. Hjorunges. Complex-Valued Matrix Derivatives. Cambridge University Press, 1st edition, 2011.

\section{Contact information}

Prof Andy Moorhouse, Acoustics Research Centre, University of Salford, Manchester, M5 4WT, UK.

Email: a.t.moorhouse@salford.ac.uk

\section{Acknowledgements}

This work was funded through the EPSRC Research Grant EP/P005489/1 Design by Science. The authors would like to acknowledge A.Clot and R.S.Langley at the University of Cambridge for their involvement in this work. 


\section{Definitions/Abbreviations}

TPA transfer path analysis

ICC interface completeness criterion, or interface completeness coefficient

FRF frequency response function 